\title{
Crushing Behavior of Thin-Walled Hexagonal Tubes with Partition Plates
}

\author{
Dai-Heng Chen and Kenichi Masuda \\ Department of Mechanical Engineering, Tokyo University of Science, Kagurazaka 1-3, Shinjuku-Ku, Tokyo 162-8601, Japan \\ Correspondence should be addressed to Dai-Heng Chen, chend@rs.kagu.tus.ac.jp
}

Received 14 January 2011; Accepted 21 February 2011

Academic Editors: N. Anifantis and P. Dineva

Copyright ( 2011 D.-H. Chen and K. Masuda. This is an open access article distributed under the Creative Commons Attribution License, which permits unrestricted use, distribution, and reproduction in any medium, provided the original work is properly cited.

\begin{abstract}
The crushing behaviour of hexagonal thin-walled tube with partition plates subjected to axial compression is studied by using finite element method. It is found that, in the crushing process, the folds, which generate along the full length of the tube, come to be crushed simultaneously and the compressive load will not descend, since the compressive load produced in the central part does not descend with the folds forming on outer walls. Therefore, in order to suppress a fluctuation of the compression load in crushing of the tube and to raise its average compression load, it is an effective method to introduce corner parts, especially corner parts where three plates intersect, in the geometry of the thin-walled tube.
\end{abstract}

\section{Introduction}

It is increasingly common in designs of thin-walled tubes to incorporate interior partition plates to impart higher energy-absorbing capacity to the tubes, which are used for crushing using the impact of collisions [1-3]. However, there have been almost no systematic studies of the properties of tubes with these partition plates. In order to avoid the Euler buckling of tubes, material should be located far from the center axis of the tube, such as hollow circular or square tubes, in order to maximize the radius of gyration $i=\sqrt{I} / A$ (where $I$ is the moment of inertia of the cross-sectional area, and $A$ is the cross-sectional area). Incorporating partition plates inside hollow members actually reduces the section radius of gyration, so that it appears to be a step backward in preventing Euler buckling. Thus, it is essential for the development of more effective energy-absorbing structures to explain the advantage imparted by adding internal partition plates to hollow structural members.

This study conducts a systematic finite element analysis and presents investigation of the hollow regular hexagonal thin-walled tubes with internal partition plates (cf. Figure 1) under axial compressive loads to investigate the role of partition plates in the crushing behavior of tubes. Also, since initial geometric imperfections exert little effect on the axial crushing process in polygonal tubes when plasticyield occurs throughout the tube cross-section [4], there was no particular emphasis on investigating initial geometric imperfections in this study. Moreover, one of the authors showed the validation of the finite element tube model [5].

\section{Numerical Analysis Method}

The general commercial FEM package, MSC/Dytran, was employed to simulate the dynamic axial crushing of a thinwalled hexagonal tube. The dimensions of the analyzed model used are shown in Figure 1, where the material density was set to $2685 \mathrm{~kg} / \mathrm{m}^{3}$. The model was collided by a rigid body with a mass of $1920 \mathrm{~kg}$ at a constant speed of $40 \mathrm{~km} / \mathrm{h}$ so as to cause axial crushing. Because the rigid body has a large mass, it continues to move at almost the same speed after the collision, throughout the crushing process. The model was discretized using four-node thickness shell elements measuring $5 \times 5 \mathrm{~mm}^{2}$ in order to ensure smooth generation of folding. The dynamic and static coefficients of friction at the boundary nodes on the top and bottom of the model were set to $\mu_{k}=0.2$ and $\mu_{s}=0.3$, respectively. The analysis is based on a dynamic explicit solution, so the time increment $d t$ must 


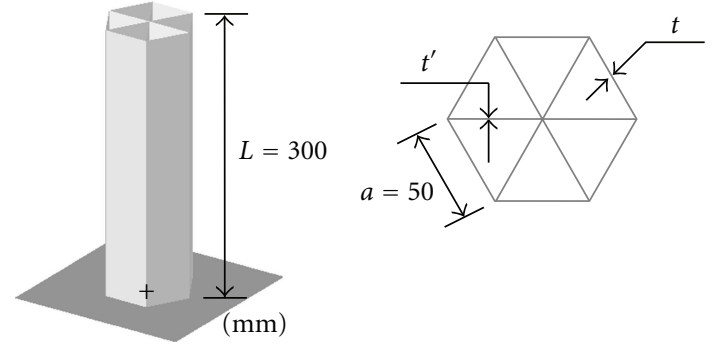

FIGURE 1: Hexagonal thin-walled tube with partition plates.

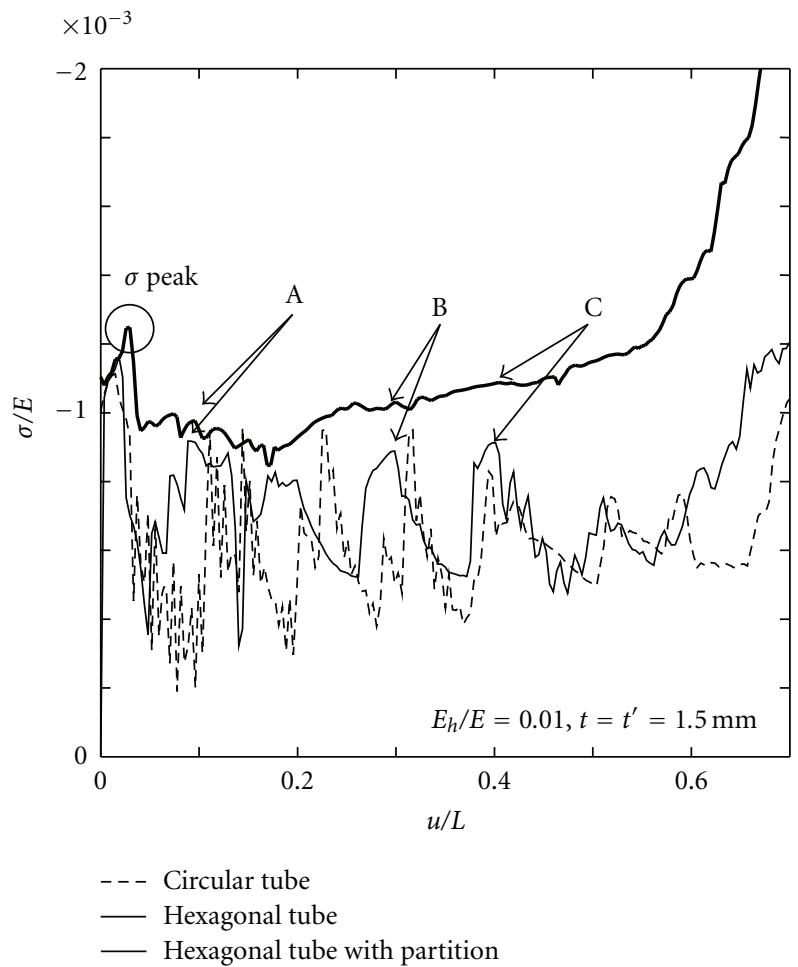

FIGURE 2: Comparison of compressive stress-crushing displacement curves between circular tube, hexagonal tube, and hexagonal tube with partition plates for $E_{h} / E=0.01$ and $t=t^{\prime}=1.5 \mathrm{~mm}$.

satisfy the following equation [6]:

$$
d t \leq \frac{L_{e}}{C}
$$

where $C$ is the propagation velocity of the elastic wave corresponding to the sound velocity and $L_{e}$ is the effective length of the element (minimum distance between element points). In addition, geometric nonlinearity was considered in present analysis through the updated Lagrangian method.

The tube material was assumed to be homogeneous and isotropic, to conform to von Mises' yield conditions and to follow the bilinear hardening curve described by the
A

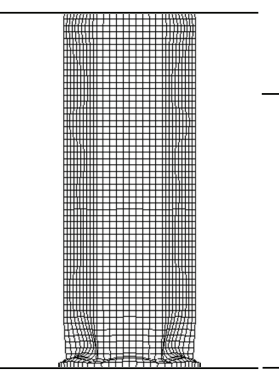

B

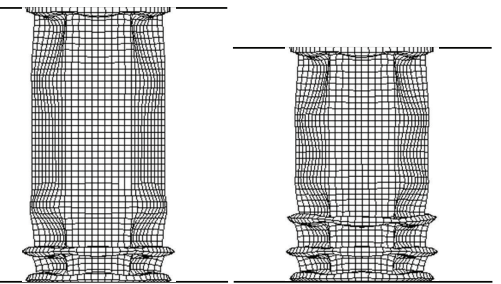

FIGURE 3: Deformation of the tube without partition plates shown in Figure 2 for crushing displacement marked by A, B, and C.
A

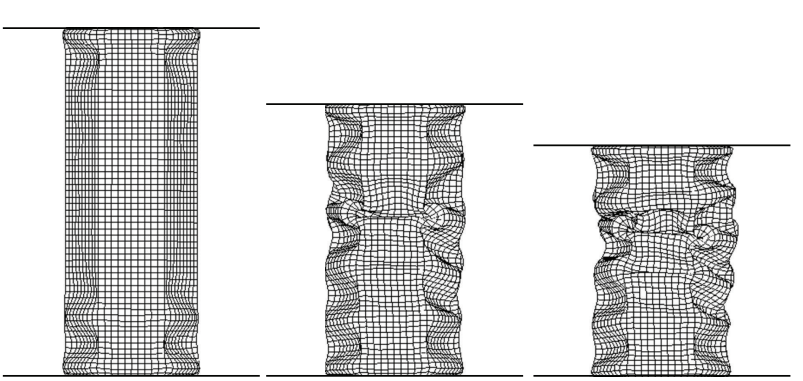

Figure 4: Deformation of the tube with partition plates shown in Figure 2 for crushing displacement marked by A, B, and C.

following stress-strain $(\sigma-\varepsilon)$ relationship in uniaxial stress state:

$$
\begin{gathered}
\sigma=E \varepsilon \quad\left(\varepsilon<\frac{\sigma_{Y}}{E}\right), \\
\sigma=\sigma_{Y}+E_{h}\left(\varepsilon-\frac{\sigma_{Y}}{E}\right) \quad\left(\varepsilon \geq \frac{\sigma_{Y}}{E}\right),
\end{gathered}
$$

where $\sigma_{Y}$ is the initial yield stress, $E$ and $E_{h}$ are the elastic modulus and strain hardening coefficient, respectively. It was assumed in this study that the material was one of the normal aluminum alloys, and the plasticity parameters were assumed to be independent of the strain rate. The material characteristics and initial values were as follows: $E=72.4 \mathrm{GPa} ; E_{h} / E=0.005,0.01,0.05 ; v=0.3$, and $\sigma_{Y}=72.4 \mathrm{MPa}$, where $v$ is the Poisson ratio.

\section{Results and Discussion}

In order to investigate the influence of partition plates on crushing behavior, the collapse mode of a regular hexagonal tube with partition plates was compared with the results obtained for a tube without partition plates.

Figure 2 shows the compressive stress and the relative crushing displacement $u / L$ of the tubes with and without internal partition plates during axial crushing. This figure also includes the compressive stress-crushing displacement curve calculated for a circular tube having the same wall thickness as the hexagonal tube and an outer diameter 


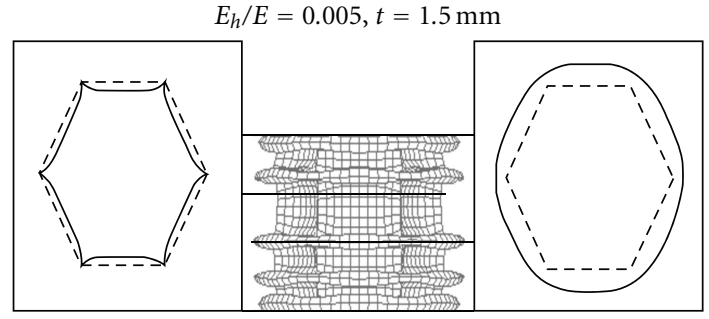

(a)

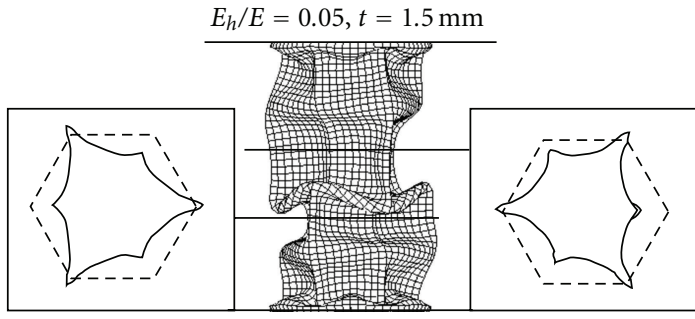

(b)

Figure 5: Cross-section of crushed tube without partition with (a) $E_{h} / E=0.005$ and $t=1.5 \mathrm{~mm}$ (b) $E_{h} / E=0.05$ and $t=1.5 \mathrm{~mm}$.

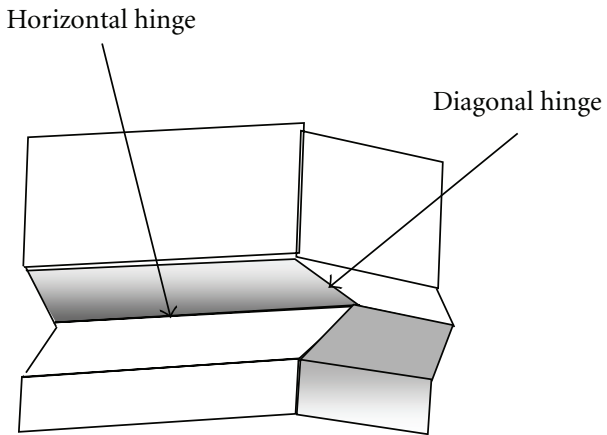

(a)

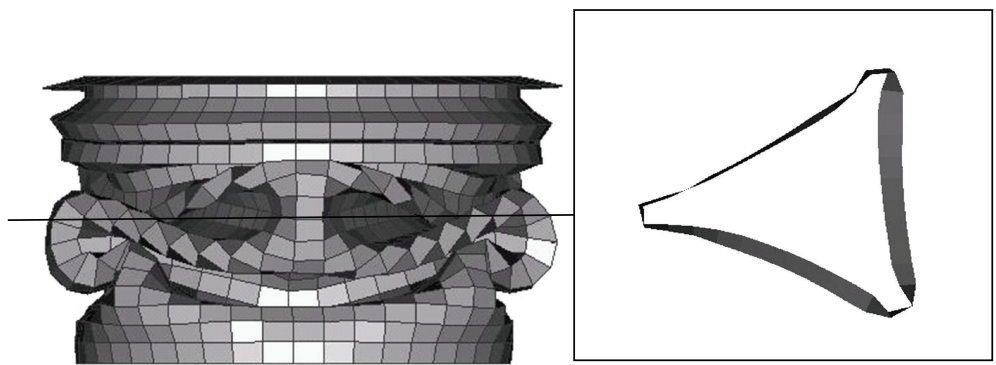

(b)

Figure 6: Collapse modes of the radial deformation: (a) inextensional-mode of square tube; (b) nonsymmetric mode of cylindrical tube.

that circumscribes the hexagonal section. In this study, the compressive stress is defined as the reaction force at the tube lower end divided by the initial cross-sectional area. The tube without partition plates behaved quite differently than the tube with partition plates. The hollow hexagonal tube exhibited periodic fluctuation in compressive stress as observed in the circular tube. The tube with partition plates displayed only very minor oscillations, increasing almost monotonically in a curve that is higher than the peak values in each periodic fluctuation of the hollow tubes. The differences in the compressive stress-crushing displacement curves were attributed to different collapse modes. Figures 3 and 4 show the deformed shapes of the tubes without and with partition plates for the points labeled $\mathrm{A}, \mathrm{B}$, and $\mathrm{C}$ in Figure 2. The tube subjected to axial compression bulged outward due to the Poisson effect, but the friction at the ends restrained the tubes from expanding at the ends. This explains why the folds appeared at the ends first, indicating the onset of buckling. However, comparing Figures 3 and 4 indicates that the tube with partition plates and the tube without partition plates clearly had different processes for the development of fold. The hollow hexagonal tube without partition plates showed the same progressive collapse mode as the circular tube; one fold generated and deformed until the fold completely collapsed, after that the next fold began to develop. In contrast, the tube with partition plates showed a simultaneous collapse mode, in which folds appeared throughout its length and all then gradually collapsed together as the crushing process progressed. This is the reason why there is almost no fluctuation in the compressive stress-crushing displacement curve of the hexagonal tube with partition plate in Figure 2.

In order to elucidate the mechanisms of simultaneous collapse mode exhibited by the tube with partition plates, next we investigate changes of the cross-section in radial direction and the vertical section in axial direction.

Figures 5(a) and 5(b) show the shapes of cross-section of the hexagonal tube without partition plates as it deforms under the conditions of $E_{h} / E=0.005$ and 0.05 , respectively, with $t=1.5 \mathrm{~mm}$. There are essentially two collapse modes for tubes without partition plates, as Figures 5(a) and 5(b) show. All the side walls of the tube in Figure 5(a) deformed in essentially the same fashion. This means that in-plane expansion occurs for all the walls of the tube. Therefore this mode is known as the "extensional mode." This corresponds exactly to the mode observed in axially symmetric cylinders. In the mode shown in Figure 5(b) however, the side walls exhibit different deformations. In a square tube, when neighboring walls show different deformation, such mode is often referred to as the inextensional mode $[7,8]$. As shown in Figure 6(a), in the inextensional mode of a square tube there usually are two features for folding of plates constituting the tube: neighboring side plates bend in alternating directions and there must also be diagonal hinge lines generated in the side plates. However, for hexagonal tube, the deformation identified in this study, shown in Figure 5(b), differs from that in the inextensional mode depicted in Figure 6(a) and appears to correspond more closely to the nonsymmetric deformation mode seen in the cylinder shown in Figure 6(b). 


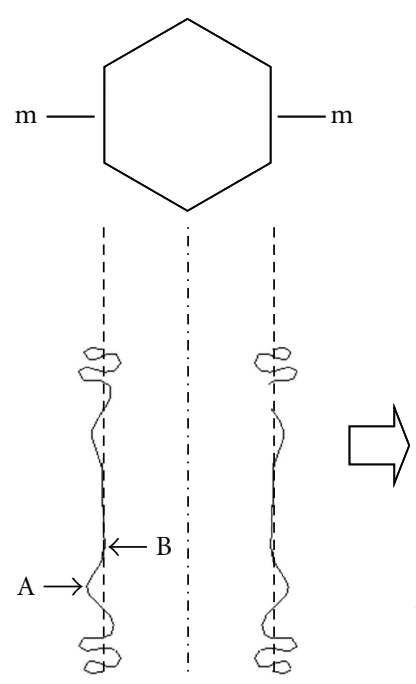

(a)

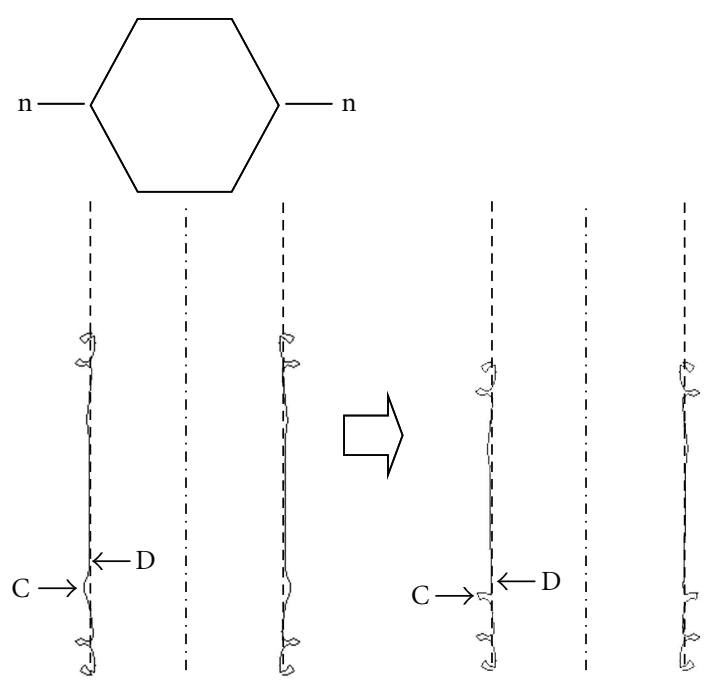

(b)

FIGURE 7: Deformed shapes of vertical section in crushing process of tube show in Figure 5(a): (a) in the middle of the outer wall (b) at the corner part.

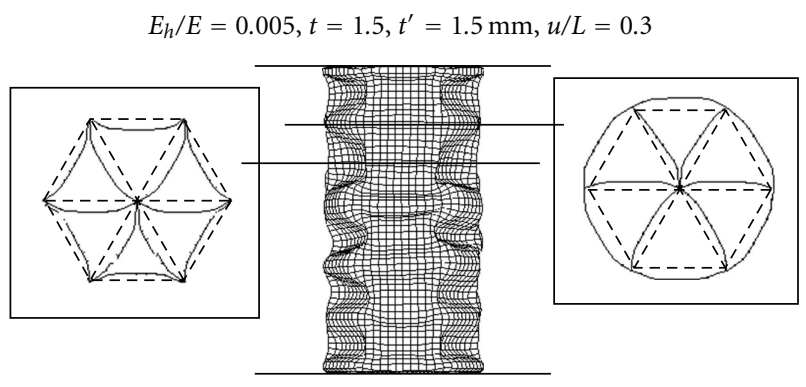

(a)

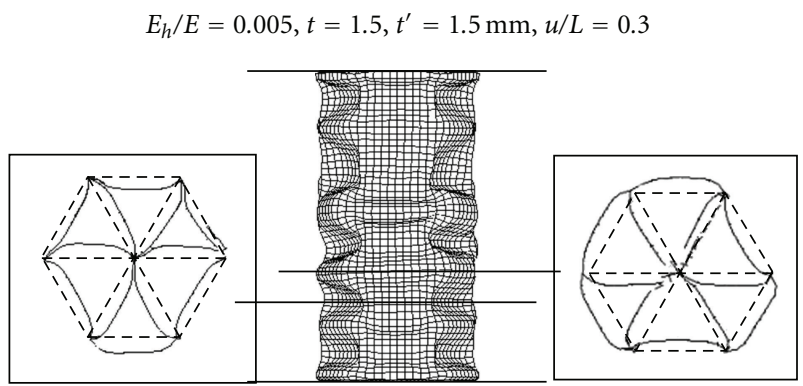

(b)

Figure 8: Deformed cross-section of crushed tube with partition with $E_{h} / E=0.005$ and $t=t^{\prime}=1.5 \mathrm{~mm}$.

Thus, the axial collapse deformation mode in a hexagonal tube without internal partition plates is closer to that of a circular tube than to that of a square tube, but, the deformation of a hexagonal tube differs from the smooth inward displacement of the wall like a circular tube, due to the presence of the vertices in the polygonal cross-section; this is clear from the inward displacement of the wall in Figure 5(a). This can be easily understood by comparing the deformations of the middle of a wall and of the vertex.
Figures 7(a) and 7(b) illustrate the deformation process of the middle of a wall (section $\mathrm{m}-\mathrm{m}$ in Figure 7) and of a corner (section $n-n$ in Figure 7 ) in the axial direction, respectively, of the tube during the axial compression depicted in Figure 5(a). The fold in the middle of the wall forms as Point A moves outward and Point B moves inward, just as occurs in a circular tube. Meanwhile at the vertex, Point $\mathrm{C}$, which is at the same height as the apex of the fold on the wall, begins to form a fold at the vertex by moving outward, but Point $\mathrm{D}$, which is at the same height as the valley of the fold on the wall, does not move inward. As a result, the cross-section adopts the star shape shown in the left side of Figure 5(a).

Figure 8 presents deformed shapes of cross-section of the hexagonal tube with partition plates at axial displacement $u / L=0.3$. Figure 8 (a) shows some resemblance to the extensional mode, in which one can see the nearly identical deformations at all its outer walls. In Figure 8(b), some outer walls displace inward and the others displace outward. However the deformation differs from the ordinary inextensional mode, because displacements are not simply in opposing directions in alternating walls. Still, no nonsymmetric deformation mode like that seen in Figure 5(b) in the hexagonal tube without partition plates was observed in the tube with partition plates. Nonsymmetric deformation mode requires the displacement of the vertices as can be seen by comparing the postdeformation cross-section with the predeformation cross-section indicated by the dashed lines in Figure 5(b). However, such displacement of the vertices is difficult for the tube with partition plates, because motion of the vertices was constrained by the partition plates. Comparison of the predeformation (dashed lines) and post-deformation cross-sections in Figure 8 shows that the vertex positions were virtually unchanged. Therefore, in the hexagonal tube without partition plates, in order to consider whether the inplane deformation occurring in addition to those bending deformations can become an essential factor, it is essential 


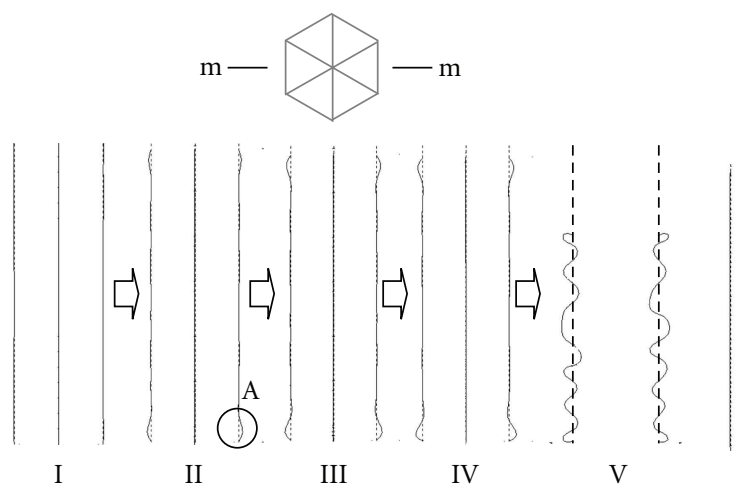

(a)

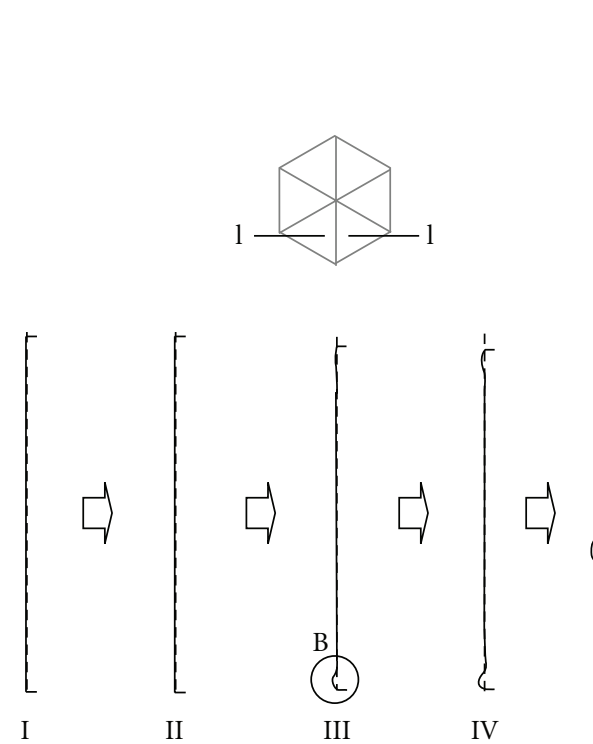

(c)

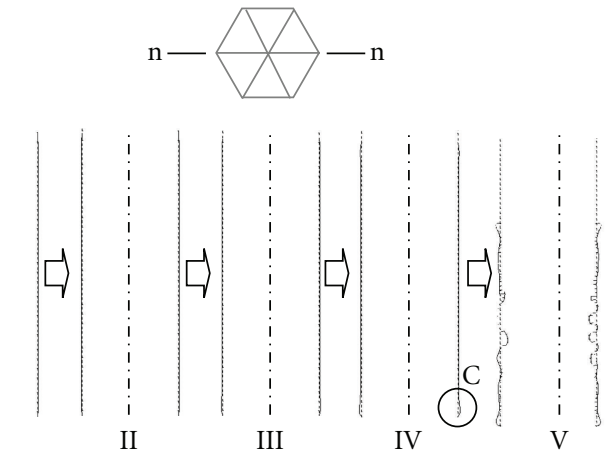

(b)

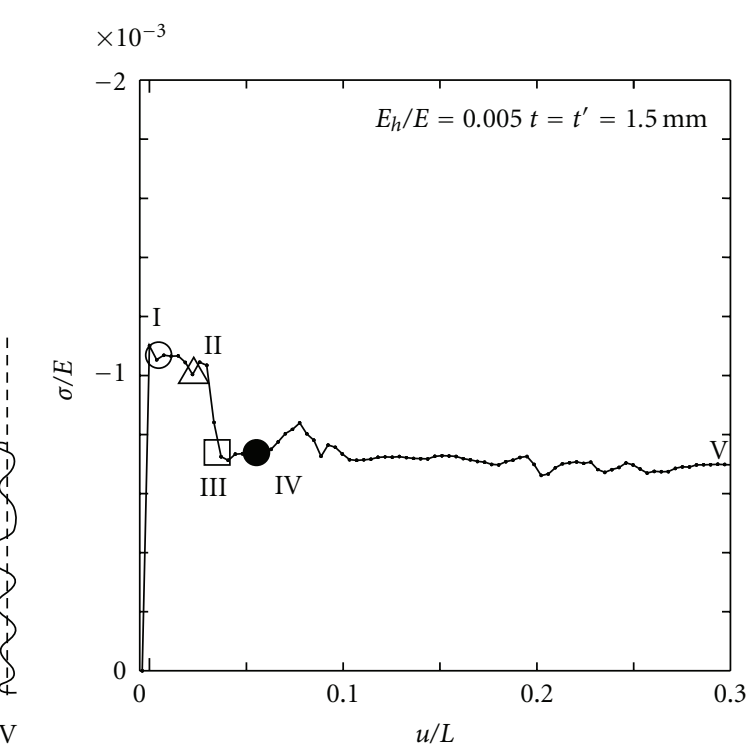

(d)

FIGURE 9: Deformed shapes of vertical section in the crushing process of a tube with partition plates: (a) section of $m$-m; (b) section of $n-n$; (c) section of l-1; (d) relation of $\sigma / E$ and $u / L$ corresponding to vertical sections shown in (a)-(c).

to find out whether the displacements of a wall and those of its neighboring walls are the same; namely, it is essential to identify whether the deformation is in the extensional mode or the inextensional mode. However, in the hexagonal tube with partition plates, the outer walls deform with two edges which are almost fixed, because the presence of the partition plates almost completely constrains the vertices of the outer walls from displacing. All the walls deform at almost the same boundaries. It is thus not necessary to distinguish whether neighboring walls displace in the same direction or not; in either case, there is bending deformation accompanying inplane deformation.

Figures 9(a), 9(b), and 9(c) show the deformed shapes in axial direction of the tube with partition plates shown in Figure 8, displaying cross-sections $1-1, m-m$, and $n-n$, with stages of collapse corresponding to I $\sim \mathrm{V}$ in Figure 9(d). Deformations of the middle of the outer walls and of the central part of the tube can be observed along section m$\mathrm{m}$, deformations of the outer vertices can be observed along section $n-n$, and deformations of the middle of the partition plates can be observed along section 1-1. The partition plates were removed from Figure 9(b) to provide a clear view of the deformation along section $n-n$ on the outer vertex; the dotdashed line in the figure does not represent the deformation of the central part of the tube. In stage V of Figure 9(a), since deformation of the central part of the tube also occurs in the direction out of the plane of $\mathrm{m}-\mathrm{m}$, deformations of the central part of the tube cannot always be observed along section $\mathrm{m}-\mathrm{m}$. Therefore, in stage $\mathrm{V}$ the central part of the tube was omitted from the Figure 9(a). The partition plates were also omitted from the deformation diagrams in Figure 10, which shows the deformation on the central part of the tube at $u / L=0.3$ from directions $\mathrm{A}$ and $\mathrm{B}$.

As can be seen in Figure 9, the initial stages I $\sim$ IV in the tube with partition plates consist of folding in the outer wall ("A" in Figure 9(a)), followed by folding in the partition plate ("B" in Figure 9(c)), and after that, folding of the outer vertex ("C" in Figure 9(b)). Even when the middle of the outer walls becomes greatly folded, as in stage IV in Figure 9(a), the central part of the tube, where all the partition plates 

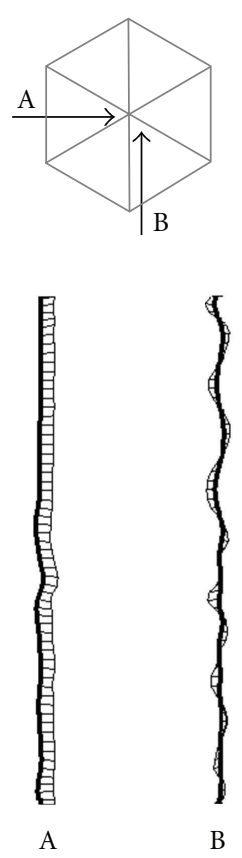

Figure 10: Deformed shapes of the central part of the tube shown in Figure 8 for $u / L=0.3$.

converge, remains almost as straight as it was before. Once crushing has progressed to a large extent, at $u / L=0.3$, folds appear throughout the lengths of the outer walls and the partition plates, as shown in Figures 9(a) and 9(c). Gentle waveforms appear in the central part of the tube, as shown in Figure 10, although these are not folded like the bends in the outer walls and partition plates. This figure depicts the deformations of the partition plate at and near the central part of the tube; the central part is indicated by the bold line in the figure.

Observation of the discrepancies between the deformation patterns of the middle of the outer wall, the middle of the partition plate, the outer vertices, and the central part of the tube shows that each of these components has a different axial compressive stress during the crushing of this tube. Figure 11 presents the distributions of the axial compressive stress in the outer wall and partition plates corresponding to the deformations at stages I $\sim$ IV in Figure 9(d) (the $\circ, \triangle, \square$, and $\bullet$ symbols in this figure correspond to those in Figure $9(\mathrm{~d})$ ). Once the outer wall generates a fold, the compressive stress decreases abruptly, while the compressive stress of the partition plates increases a little (note the $\Delta$ symbols in Figure 11(b)). After that, folds also occur in the partition plates, so the compressive stress of the partition plates decreases, but by a lower amount than that at the outer wall. The central part of the tube remains straight, so the axial compressive stress actually increases in that vicinity (near $s / a=1.0$ in Figure 11(b)). Therefore, the large compressive stress is needed to continue the folding, and the collapse mode for the tube with partition plates is not progressive collapse mode, in which the fold is formed one after another, but simultaneous collapse mode, in which all of the folds throughout the length of the tube grow and collapse together. The axial compressive stress does not diminish during this process. In contrast, a tube without partition plates shows a progressive collapse mode. The axial compressive stress decreases with the appearance of the first fold, and continues to diminish with the development of collapse. As a result, the deformation is concentrated at the location where the fold occurred, and crushing continues till completion at the generated fold before the next location develops a fold. Therefore, the compressive stress fluctuates with the fold forming one after another during the collapse process.

The above investigation demonstrates that in the vertices, especially three-wall vertices, generation of folds is restrained and a higher compressive stress than in walls is yielded. This suppresses fluctuations in the compressive stress, and thus the average compressive stress increases due to this mechanism. The vertices play a critical role in the collapse of this kind of structure. Therefore, one strategy for enhancing the ability of thin-walled tubes to absorb crushing energy is to use tubes with multiple vertices. Figure 12 shows examples of such kinds of tubes that were investigated for this property. Figure 12(a) presents the compressive stresses for square tubes with two configurations of internal partition plates. Type A had more vertices than Type B and consequently showed a higher axial compressive stress. Figure 12(b) compares the compressive stress in hexagonal tubes with two configurations of partition plates, one the same as that described in this paper and the other with a small cylindrical tube structure located at the centerline of the tube in order to increase the cross-sectional area of the centerline of the tube. This demonstrates that increasing the cross-sectional area of the central part of the tube, which supports a high stress, further increases the energy-absorbing capacity of the tube.

\section{Conclusions}

In this paper, we investigated of the hexagonal thin-walled tube with partition plates subjected to axial compression by using finite element method. The following results became apparent during this investigation.

(1) Even when the middle of the outer walls becomes greatly folded for the hexagonal tube with partition plates subjected to axial compression, the axial compression stress does not descend at the central part. Therefore, the larger compressive stress is needed to continue the folding.

(2) Due to conclusion (1), the collapse mode for the tube with partition plates is not progressive collapse mode, in which the fold is formed one after another, but simultaneous collapse mode, in which all of the folds throughout the length of the tube grow and collapse together. The axial compressive stress does not diminish during this process.

(3) In order to suppress a fluctuation of the compression stress in crushing of tube and to raise its average compression stress, it is an effective method to 


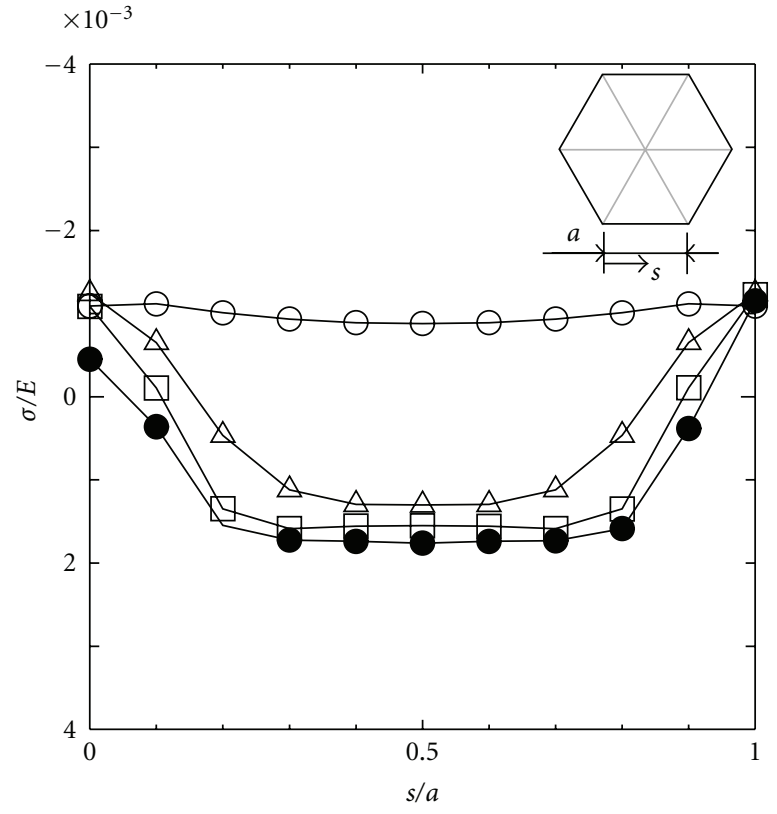

(a)

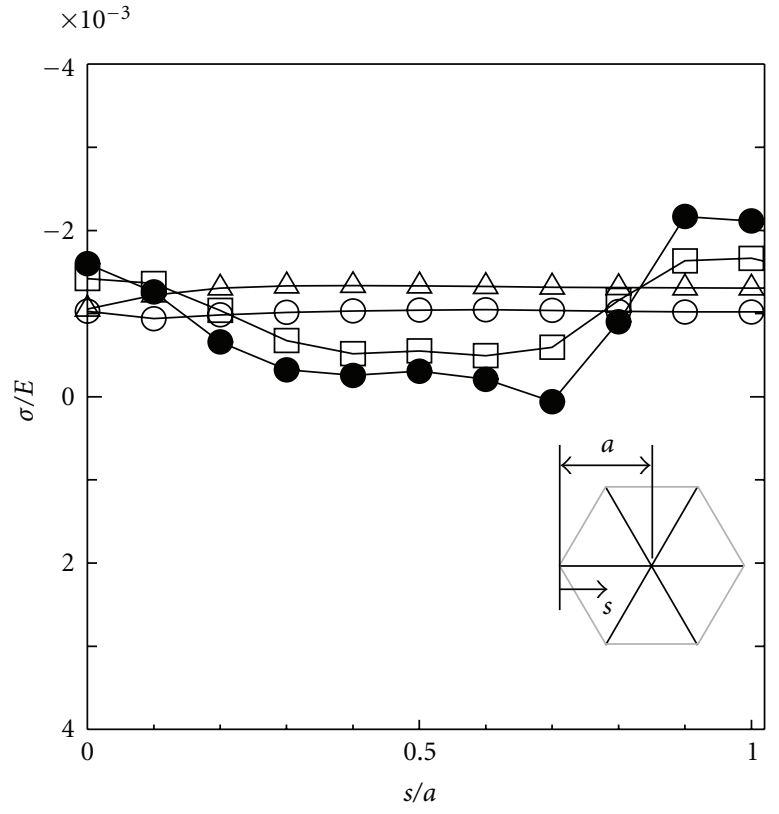

(b)

FIgURE 11: Axial stress distribution of the tube shown in Figure 9: (a) outer wall; (b) partition plate.

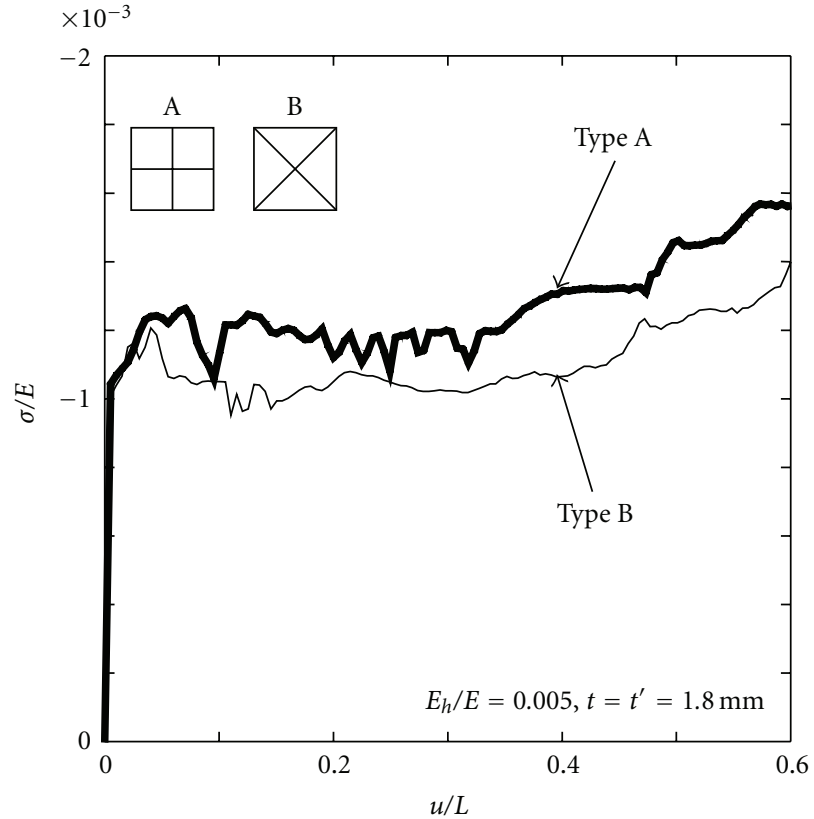

(a)

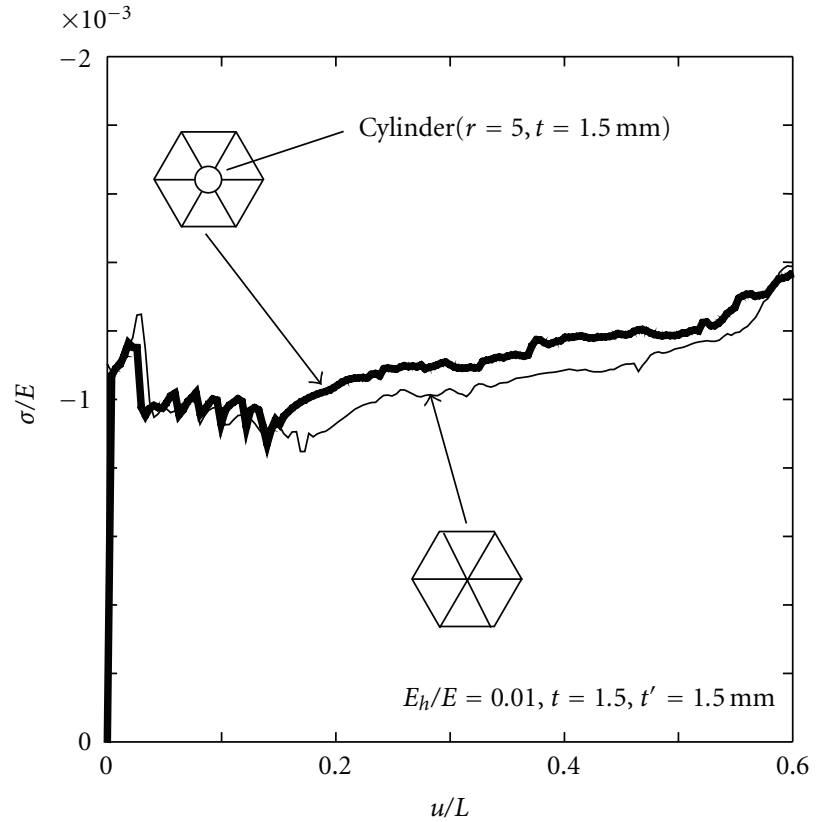

(b)

Figure 12: Relations of $\sigma / E$ and $u / L$ : (a) for square tube with two configurations of internal partition plates with $E_{h} / E=0.005$ and $t=t^{\prime}=$ $1.8 \mathrm{~mm}$; (b) for hexagonal tube with two configurations of internal partition plates with $E_{h} / E=0.01$ and $t=t^{\prime}=1.8 \mathrm{~mm}$.

introduce vertices, especially three-wall vertices, in the geometry of the thin-walled tube.

\section{References}

[1] T. Yamada et al., Analysis of Buckling Behaviour of Aluminum Structure for Crash Absorbing, SAE International, 2004.
[2] Y. Estrin et al., "Materials with novel architectonics: of interlocked elements," in Proceedings of the IUTAM Symposium on Analytical and Computational Fracture Mechanics of Non-Homogeneous Materials, pp. 51-55, 2002.

[3] T. Yamao, K. Iwatsubo, T. Yamamuro, M. Ogushi, and S. Matsumura, "Steel bridge piers with inner cruciform plates under cyclic loading," Thin-Walled Structures, vol. 40, no. 2, pp. 183-197, 1984. 
[4] B. C. Batterman, "Plastic buckling of axially compressed cylindrical shells," AIAA Journal, vol. 3, pp. 316-325, 1965.

[5] D. H. Chen and K. Ushijima, "Estimation of the initial peak load for circular tubes subjected to axial impact," Thin-Walled Structures. In press.

[6] MSC, Dytran User's Guide, 2003.

[7] W. Abramowicz and N. Jones, "Dynamic axial crushing of square tubes," International Journal of Impact Engineering, vol. 2, no. 2, pp. 179-208, 1984.

[8] W. Johnson, "Inextensional collapse of thin-walled tubes under axial compression," Journal of Strain Analysis, vol. 12, no. 4, pp. 317-330, 1977. 

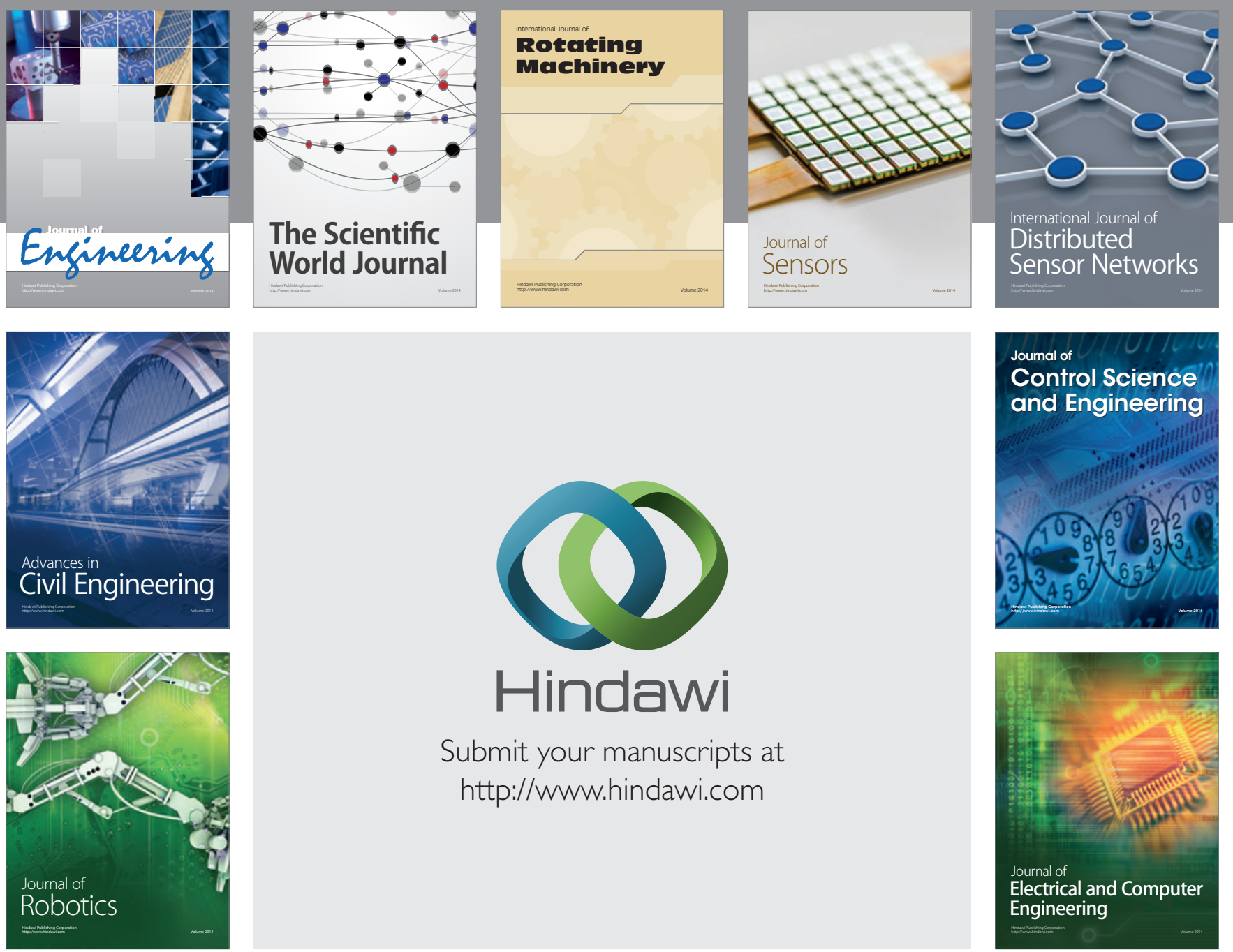

Submit your manuscripts at

http://www.hindawi.com
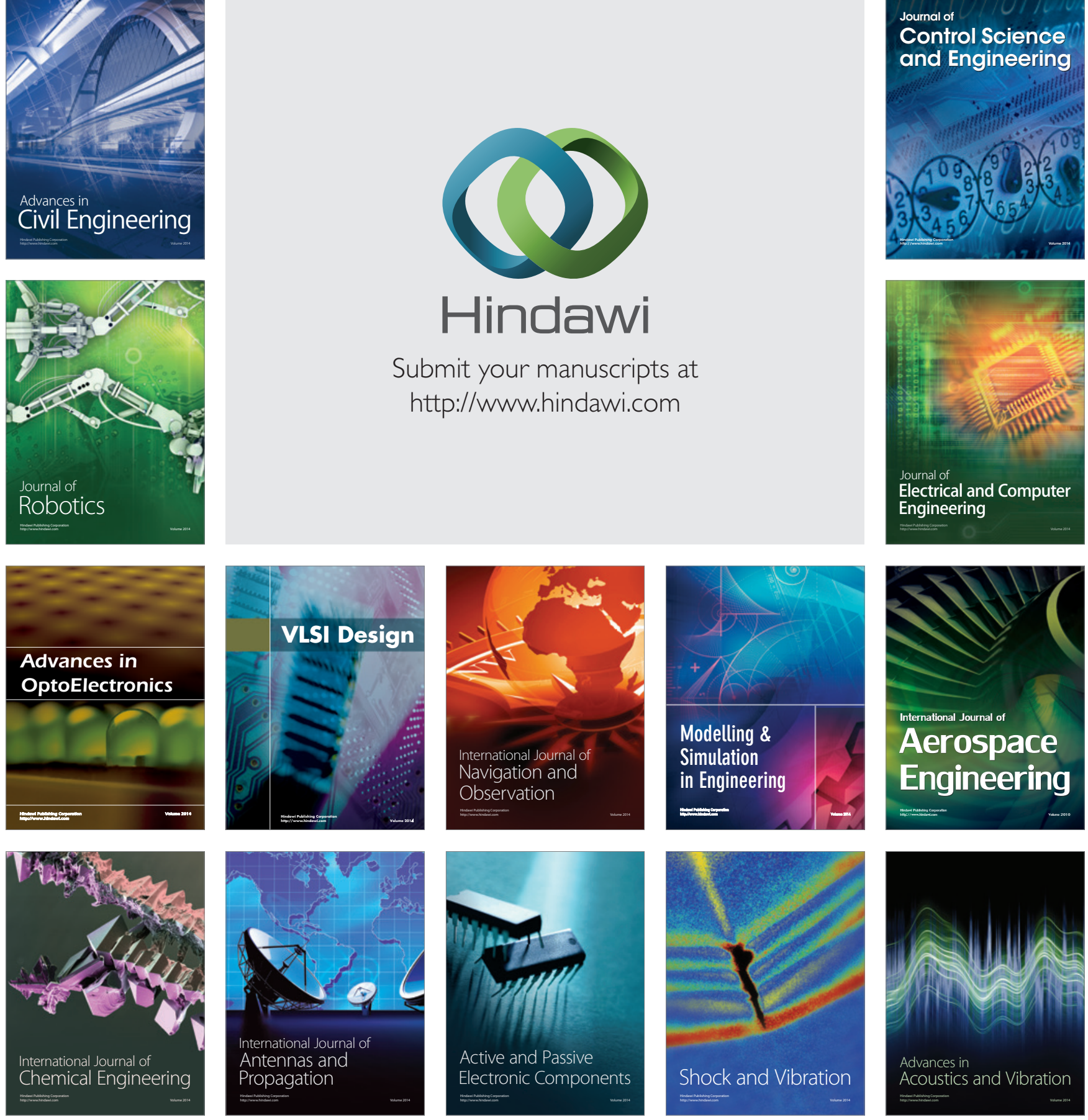